\title{
Improving mentoring through a blend of novice and peer, near peer and group mentoring: a commentary
}

\section{Commentary}

Mentoring is increasingly seen as a means of providing appropriate, personalised, timely and holistic support for learners. ${ }^{1-5}$ Recent reviews suggest that a particularly effective means of providing such support lies in novice mentoring or mentoring between senior clinicians and medical students or junior doctors. However shortages of mentors, time constraints both on the part of mentees and mentors and concerns about the timeliness of mentoring support have raised concerns as to the viability of novice mentoring on a larger scale.

Our experiences with mentoring in the Palliative Medicine Initiative (PMI) run by the Division of Palliative Medicine at the National Cancer Centre Singapore offers a potential solution for this problem. Here novice mentoring is coupled with near peer and peer mentoring to provide timely mentoring support (Figure 1).

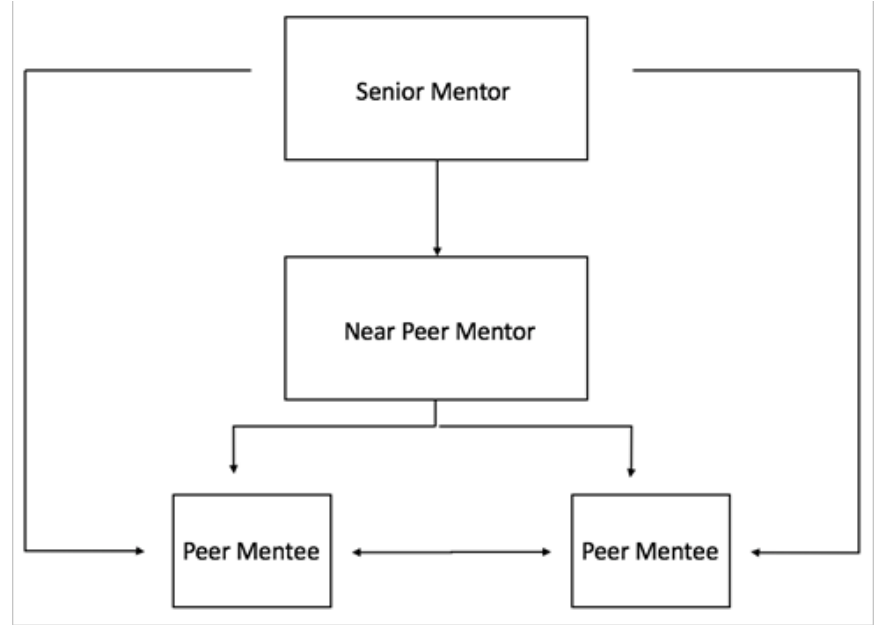

Figure I Mentoring in the palliative medicine initiative that sees mentoring support also provided through phone contact, email and text and whatsapp messaginga.

Peer mentorship is widely employed by various medical institutions and can take the form of peer, near peer and group mentoring. Junior doctors mentor each other and or medical students in groups or on a one to one basis guided by a senior mentor. This achieves the dual function of encouraging peers to share resources and help each other, while providing a guide who crucially possesses the necessary professional, academic and psychosocial experience that can translate into practical advice and help. Near peer mentorship from housemen to students for example has proven more experientially relevant to the struggles of the student. Kman et al. ${ }^{6}$ notes that peer mentorship "avoids the common mentoring pitfall known as the "authority gradient" in which the gap between mentor and mentee leads to an ineffective relationship". 7,8 Also significantly noted that peer mentorship usually evolved into a connection that more resembled a friendship than a mere working relationship which has been found to sustain effective mentoring relationships. ${ }^{4}$ Peer and near peer mentorship allows students to appreciate the value of working together as a team,

\author{
Volume 5 Issue I - 2018
}

\author{
Quek Qian Min Simone, Ong Zheng Xuan \\ Department of Nursing, National University of Singapore, \\ Singapore
}

Correspondence: Quek Qian Min Simone, Department of Nursing, National University of Singapore, Singapore, Email a0116895@u.nus.edu

Received: July 04, 2017 | Published: February 12, 2018

whether it is with peers or a senior mentor, to achieve both overall and individual success. Similar struggles, interests and aspirations discussed forges bonds that are gradually strengthened over the period of mentorship. ${ }^{6}$ The process is also mutually beneficial with senior members of the near peer mentoring dyad "developed essential attributes of time management, communication and leadership for mentors". ${ }^{7}$ The combination of group, near peer, peer and novice mentoring also rewards mentors with "the recognition... the teaching can be enjoyable, rewarding and beneficial to them" which in turn urges them to continue capitalizing on their strengths and improve on their weaknesses.

Overall the PMI mentoring model also creates an environment in which experiential learning empowers mentors to make a difference and witness that change for themselves. We believe the cycle of affirmation constructed between mentor and mentee in PMI style mentoring creates a positive culture of constant learning and improvement, which may continue to have an extensive impact on the individual as well as future generations of medical students even as they prepare to enter the medical profession.

\section{Acknowledgments}

None.

\section{Conflict of interest}

The author declares no conflict of interest.

\section{References}

1. Wahab MT, Wu JT, Ikbal MF, et al. Creating effective interprofessional mentoring relationships in palliative care -lessons from medicine, nursing, surgery, and social work. J Palliat Care Med. 2016;6:290.

2. Yeam CT, Loo TWW, Ee HFM, et al. An evidence-based evaluation of prevailing learning theories on mentoring in palliative medicine. Palliat Med Care. 2016;3:1-7.

3. Loo TWW, Ikbal MF, Wu JT, et al. Towards a practice guided evidence based theory of mentoring in palliative care. $J$ Palliat Care Med. 2017;7:296.

4. Sng JH, Pei Y, Toh YP, et al. Mentoring relationships between senior physicians and junior doctors and/or medical students: a thematic review. Med Teach. 2017;39(8):866-875. 
5. Toh YP, Lam B, Soo J, et al. Developing palliative care physicians through mentoring relationships. Palliat Med Care. 2017;4:1-6.

6. Kman NE, Bernard AW, Khandelwal S, et al. A tiered mentorship program improves number of students with an identified mentor. Teach Learn Med. 2013;25(4):319-325.

7. Tran K, Tran GT, Fuller R. West yorkshire mentor scheme: teaching and development. Clin Teach. 2014;11(1):48-52.
8. Kosoko Lasaki O, Sonnino RE, et al. Mentoring for women and underrepresented minority faculty and students: experience at two institutions of higher education. J Natl Med Assoc. 2006;98(9):14491459.

9. Wu JT, Wahab MT, Ikbal MF, et al. Toward an interprofessional mentoring program in palliative care-a review of undergraduate and postgraduate mentoring in medicine, nursing, surgery and social work. $J$ Palliat Care Med. 2016;6:292. 